\title{
New Delay-Dependent Robust Stability Criterion for LPD Discrete-Time Systems with Interval Time-Varying Delays
}

\author{
Narongsak Yotha ${ }^{1}$ and Kanit Mukdasai ${ }^{2,3}$ \\ ${ }^{1}$ Department of Mathematics, Faculty of Science, Chiang Mai University, Chiang Mai 50200, Thailand \\ ${ }^{2}$ Department of Mathematics, Faculty of Science, Khon Kaen University, Khon Kaen 40002, Thailand \\ ${ }^{3}$ Centre of Excellence in Mathematics, CHE, Si Ayutthaya Road, Bangkok 10400, Thailand \\ Correspondence should be addressed to Kanit Mukdasai; kanit@kku.ac.th
}

Received 18 October 2012; Accepted 13 January 2013

Academic Editor: Xiaohui Liu

Copyright (C) 2013 N. Yotha and K. Mukdasai. This is an open access article distributed under the Creative Commons Attribution License, which permits unrestricted use, distribution, and reproduction in any medium, provided the original work is properly cited.

\begin{abstract}
This paper investigates the problem of robust stability for linear parameter-dependent (LPD) discrete-time systems with interval time-varying delays. Based on the combination of model transformation, utilization of zero equation, and parameter-dependent Lyapunov-Krasovskii functional, new delay-dependent robust stability conditions are obtained and formulated in terms of linear matrix inequalities (LMIs). Numerical examples are given to demonstrate the effectiveness and less conservativeness of the proposed methods.
\end{abstract}

\section{Introduction}

Systems with time delay exist in many fields such as electric systems, chemical processes systems, networked control systems, telecommunication systems, and economical systems. Over the past decades, the problem of robust stability analysis for uncertain systems with time delay has been widely investigated by many researchers. Commonly, stability criteria for uncertain systems with time delay are generally divided into two classes: a delay-independent one and a delay-dependent one. The delay-independent stability criteria tends to be more conservative, especially for a small size delay; such criteria do not give any information on the size of delay. On the other hand, delay-dependent stability criteria are concerned with the size of delay and usually provide a maximal delay size.

Discrete-time systems with state delay have strong background in engineering applications, among which networkbased control has been well recognized to be a typical example. If the delay is constant in discrete systems, one can transform a delayed system into a delay-free one by using state augmentation techniques. However, when the delay is large, the augmented system will become much complex and thus difficult to analyze and synthesize [1]. In recent years, robust stability analysis of continuous-time and discrete-time systems subject to time-invariant parametric uncertainty has received considerable attention. An important class of linear timeinvariant parametric uncertain system is a linear parameterdependent (LPD) system in which the uncertain state matrices are in the polytope consisting of all convex combination of known matrices. To address this problem, several results have been obtained in terms of sufficient (or necessary and sufficient) conditions, see [1-24] and references cited therein. Most of these conditions have been obtained via the Lyapunov theory approaches in which the parameter-dependent Lyapunov functions have been employed. These conditions are always expressed in terms of LMIs which can be solved numerically by using available tools such as the LMI Toolbox in MATLAB. Recently, delay-dependent robust stability criteria for LPD continuous-time systems with time delay have been taken into consideration. Sufficient conditions for robust stability of time-delay systems have been presented via Lyapunov approaches $[8,16,21]$. However, much attention has been focused on the problem of robust stability analysis for LPD discrete-time systems with time delay $[10,13,22]$.

In this paper, we focus on the delay-dependent robust stability criterion for LPD discrete-time systems with interval time-varying delays. Based on the combination of model 
transformation, utilization of zero equation, and parameterdependent Lyapunov functional, new delay-dependent robust stability conditions are obtained and formulated in terms of linear matrix inequalities (LMIs). Finally, numerical examples are given to illustrate that the resulting criterion outperforms the existing stability condition.

\section{Problem Formulation and Preliminaries}

We introduce some notations, definitions, and propositions that will be used throughout the paper. $\mathbb{Z}^{+}$denotes the set of nonnegative integer numbers; $\mathbb{R}^{n}$ denotes the $n$-dimensional space with the vector norm $\|\cdot\| ;\|x\|$ denotes the Euclidean vector norm of $x \in \mathbb{R}^{n}$, that is, $\|x\|^{2}=x^{T} x ; M^{n \times r}$ denotes the space of all real matrices of $(n \times r)$-dimensions; $A^{T}$ denotes the transpose of the matrix $A ; A$ is symmetric if $A=A^{T} ; I$ denotes the identity matrix; $\lambda(A)$ denotes the set of all eigenvalues of $A ; \lambda_{\max }(A)=\max \{\operatorname{Re} \lambda: \lambda \in \lambda(A)\} ; \lambda_{\min }(A)=$ $\min \{\operatorname{Re} \lambda: \lambda \in \lambda(A)\} ; \lambda_{\max }(A(\alpha))=\max \left\{\lambda_{\max }\left(A_{i}\right): i=\right.$ $1,2, \ldots, N\} ; \lambda_{\min }(A(\alpha))=\min \left\{\lambda_{\min }\left(A_{i}\right): i=1,2, \ldots, N\right\}$; matrix $A$ is called a semipositive definite $(A \geq 0)$ if $x^{T} A x \geq 0$, for all $x \in \mathbb{R}^{n} ; A$ is a positive definite $(A>0)$ if $x^{T} A x>0$ for all $x \neq 0$; matrix $B$ is called a seminegative definite $(B \leq 0)$ if $x^{T} B x \leq 0$, for all $x \in \mathbb{R}^{n} ; B$ is a negative definite $(B<0)$ if $x^{T} B x<0$ for all $x \neq 0 ; A>B$ means $A-B>0 ; A \geq B$ means $A-B \geq 0 ; *$ represents the elements below the main diagonal of a symmetric matrix.

Consider the following uncertain LPD discrete-time system with interval time-varying delays of the form

$$
\begin{aligned}
& x(k+1)=A(\alpha) x(k)+B(\alpha) x(k-h(k)), \\
& x(s)=\phi(s), \quad s \in\left\{-h_{2},-h_{2}+1, \ldots,-1,0,\right\},
\end{aligned}
$$

where $k \in \mathbb{Z}^{+}, x(k) \in \mathbb{R}^{n}$ is the system state and $\phi(s)$ is an initial value at $s . A(\alpha), B(\alpha) \in M^{n \times n}$ are uncertain matrices belonging to the polytope of the form

$$
\begin{array}{r}
A(\alpha)=\sum_{i=1}^{N} \alpha_{i} A_{i}, \quad B(\alpha)=\sum_{i=1}^{N} \alpha_{i} B_{i}, \\
\sum_{i=1}^{N} \alpha_{i}=1, \quad \alpha_{i} \geq 0, \quad A_{i}, B_{i} \in M^{n \times n}, i=1, \ldots, N .
\end{array}
$$

In addition, we assume that the time-varying delay $h(k)$ is upper and lower bounded. It satisfies the following assumption of the form

$$
0<h_{1} \leq h(k) \leq h_{2},
$$

where $h_{1}$ and $h_{2}$ are known positive integers.

Definition 1 (see [19]). The system (1) is said to be robustly stable if there exists a positive definite function $V(k, x(k))$ : $\mathbb{Z}^{+} \times \mathbb{R}^{n} \rightarrow \mathbb{R}$ such that

$$
\Delta V(k, x(k))=V(k+1, x(k+1))-V(k, x(k))<0,
$$

along any trajectory of the solution of the system (1).
Definition 2 (see [19]). The system (1) when $A(\alpha)=A, B(\alpha)=$ $B$, and $A, B \in M^{n \times n}$ is said to be asymptotically stable if there exists a positive definite function $V(k, x(k)): \mathbb{Z}^{+} \times \mathbb{R}^{n} \rightarrow \mathbb{R}$ such that

$$
\Delta V(k, x(k))=V(k+1, x(k+1))-V(k, x(k))<0,
$$

along any trajectory of the solution of the system (1) when $A(\alpha)=A, B(\alpha)=B$.

Proposition 3 ([7, the Schur complement lemma]). Given constant symmetric matrices $X, Y$, and $Z$ of appropriate dimensions with $Y>0$, then $X+Z^{T} Y^{-1} Z<0$ if and only if

$$
\left(\begin{array}{cc}
X & Z^{T} \\
Z & -Y
\end{array}\right)<0 \quad \text { or } \quad\left(\begin{array}{cc}
-Y & Z \\
Z^{T} & X
\end{array}\right)<0
$$

Proposition 4 (see [9]). For any constant matrix $W \in$ $M^{n \times n}, W=W^{T}>0$, two integers $r_{M}$ and $r_{m}$ satisfying $r_{M} \geq r_{m}$, and vector function $x:\left[r_{m}, r_{M}\right] \rightarrow \mathbb{R}^{n}$, the following inequality holds:

$$
\left(\sum_{i=r_{m}}^{r_{M}} x(i)\right)^{T} W\left(\sum_{i=r_{m}}^{r_{M}} x(i)\right) \leq\left(r_{M}-r_{m}+1\right) \sum_{i=r_{m}}^{r_{M}} x^{T}(i) W x(i)
$$

Rewrite the system (1) in the following system:

$$
\begin{gathered}
x(k+1)=x(k)+y(k), \\
y(k)=[A(\alpha)+B(\alpha)-I] x(k)-B(\alpha) \sum_{i=k-h(k)}^{k-1} y(i) .
\end{gathered}
$$

\section{Robust Stability Conditions}

In this section, we study the robust stability criteria for the system (1) by using the combination of model transformation, the linear matrix inequality (LMI) technique, and the Lyapunov method. We introduce the following notations for later use:

$$
\begin{aligned}
C_{j}(\alpha)=\sum_{i=1}^{N} \alpha_{i} C_{i}^{j}, & D_{j}(\alpha)=\sum_{i=1}^{N} \alpha_{i} D_{i}^{j}, \\
E_{j}(\alpha)=\sum_{i=1}^{N} \alpha_{i} E_{i}^{j}, & G_{j}(\alpha)=\sum_{i=1}^{N} \alpha_{i} G_{i}^{j}, \\
L_{j}(\alpha)=\sum_{i=1}^{N} \alpha_{i} L_{i}^{j}, & P(\alpha)=\sum_{i=1}^{N} \alpha_{i} P_{i}, \\
Q(\alpha)=\sum_{i=1}^{N} \alpha_{i} Q_{i}, & R(\alpha)=\sum_{i=1}^{N} \alpha_{i} R_{i},
\end{aligned}
$$




$$
\begin{aligned}
& S(\alpha)=\sum_{i=1}^{N} \alpha_{i} S_{i}, \quad T(\alpha)=\sum_{i=1}^{N} \alpha_{i} T_{i}, \\
& U(\alpha)=\sum_{i=1}^{N} \alpha_{i} U_{i}, \quad V(\alpha)=\sum_{i=1}^{N} \alpha_{i} V_{i} \\
& W(\alpha)=\sum_{i=1}^{N} \alpha_{i} W_{i}, \quad X(\alpha)=\sum_{i=1}^{N} \alpha_{i} X_{i} \\
& Y(\alpha)=\sum_{i=1}^{N} \alpha_{i} Y_{i}, \quad Z(\alpha)=\sum_{i=1}^{N} \alpha_{i} Z_{i} \\
& \sum_{i=1}^{N} \alpha_{i}=1, \alpha_{i} \geq 0 \\
& C_{i}^{j}, D_{i}^{j}, E_{i}^{j}, G_{i}^{j}, L_{i}^{j}, P_{i}, Q_{i}, R_{i}, S_{i}, T_{i}, U_{i}, \\
& V_{i}, W_{i}, X_{i}, Y_{i}, Z_{i} \in M^{n \times n} \\
& j=1,2,3, i=1,2, \ldots, N, \rho=h_{2}-h_{1} . \\
& \prod_{i, j}=\left[\begin{array}{ccccccccc}
\Sigma_{i, j}^{11} & \Sigma_{i, j}^{12} & \Sigma_{i, j}^{13} & \Sigma_{i, j}^{14} & \Sigma_{i, j}^{15} & \Sigma_{i}^{16} & \Sigma_{i, j}^{17} & \Sigma_{i, j}^{18} & 0 \\
* & \Sigma_{i, j}^{22} & 0 & 0 & 0 & 0 & 0 & \Sigma_{i, j}^{28} & 0 \\
* & * & \Sigma_{i}^{33} & \Sigma_{i}^{34} & 0 & \Sigma_{i, j}^{36} & 0 & 0 & \Sigma_{i, j}^{18} \\
* & * & * & \Sigma_{i}^{44} & 0 & 0 & \Sigma_{i, j}^{47} & 0 & \Sigma_{i, j}^{18} \\
* & * & * & * & \Sigma_{i}^{55} & 0 & 0 & \Sigma_{i, j}^{18} & 0 \\
* & * & * & * & * & \Sigma_{i}^{66} & 0 & 0 & 0 \\
* & * & * & * & * & * & \Sigma_{i, j}^{77} & 0 & 0 \\
* & * & * & * & * & * & * & \Sigma_{i, j}^{88} & 0 \\
* & * & * & * & * & * & * & * & \Sigma_{i, j}^{99}
\end{array}\right],
\end{aligned}
$$

where

$$
\begin{aligned}
\Sigma_{i, j}^{11}= & Q_{i}+R_{i}+T_{i}+h_{1} M_{i}+N_{i}+N_{i}^{T}+h_{2} K_{i}+L_{i}+L_{i}^{T} \\
& +C_{i}^{1}+C_{i}^{1^{T}}+D_{i}^{1}+D_{i}^{1^{T}}+E_{i}^{1}+E_{i}^{1^{T}}+L_{i}^{2^{T}} A_{j} \\
& +A_{i}^{T} L_{j}^{2}+L_{i}^{2^{T}} B_{j}+B_{i}^{T} L_{j}^{2}-L_{i}^{2}-L_{i}^{2^{T}}, \\
\Sigma_{i, j}^{12}= & A_{i}^{T} L_{j}^{1}+B_{i}^{T} L_{j}^{1}-L_{i}^{1}-L_{i}^{2^{T}}+P_{i}, \\
\Sigma_{i}^{13}= & C_{i}^{2}-N_{i}-C_{i}^{1^{T}}, \quad \Sigma_{i}^{14}=D_{i}^{2}-L_{i}-D_{i}^{1^{T}}, \\
\Sigma_{i}^{15}= & -E_{i}^{1^{T}}+E_{i}^{2}, \quad \Sigma_{i}^{16}=-C_{i}^{1 T}+C_{i}^{3}, \\
\Sigma_{i}^{17}= & -D_{i}^{1^{T}}+D_{i}^{3}, \quad \\
\sum_{i, j}^{18}= & -E_{i}^{1^{T}}+E_{i}^{3}-L_{i}^{2^{T}} B_{j}+A_{i}^{T} L_{j}^{3}+B_{i}^{T} L_{j}^{3}-L_{i}^{3}, \\
\Sigma_{i}^{22}= & P_{i}+h_{1}^{2} U_{i}+h_{2}^{2} V_{i}+h_{2}^{2} W_{i}+\rho^{2} X_{i}+h_{1} Y_{i} \\
& +h_{2} Z_{i}-L_{i}^{1}-L_{i}^{1^{T}}, \quad \sum_{i, j}^{28}=-L_{i}^{1^{T}} B_{j}-L_{i}^{3},
\end{aligned}
$$

$$
\begin{aligned}
& \Sigma_{i}^{33}=-Q_{i}+S_{i}-C_{i}^{2}-C_{i}^{2^{T}}+G_{i}^{1}+G_{i}^{1^{T}} \\
& \Sigma_{i}^{34}=G_{i}^{2}-G_{i}^{1^{T}}, \quad \Sigma_{i}^{36}=-C_{i}^{3}-C_{i}^{2^{T}}, \\
& \Sigma_{i}^{39}=G_{i}^{3}-G_{i}^{1^{T}}, \quad \Sigma_{i}^{44}=-R_{i}-S_{i}-D_{i}^{2}-D_{i}^{2^{T}}-G_{i}^{2}-G_{i}^{2^{T}}, \\
& \Sigma_{i}^{47}=-D_{i}^{3}-D_{i}^{2^{T}}, \quad \Sigma_{i}^{49}=-G_{i}^{3}-G_{i}^{2^{T}}, \\
& \Sigma_{i}^{55}=-T_{i}-E_{i}^{2}-E_{i}^{2^{T}}, \quad \Sigma_{i}^{58}=-E_{i}^{3}-E_{i}^{2^{T}}, \\
& \Sigma_{i}^{66}=-U_{i}-C_{i}^{3}-C_{i}^{3^{T}}, \quad \Sigma_{i}^{77}=-V_{i}-D_{i}^{3}-D_{i}^{3^{T}}, \\
& \Sigma_{i, j}^{88}=-W_{i}-E_{i}^{3}-E_{i}^{3^{T}}-L_{i}^{3^{T}} B_{j}-B_{i}^{T} L_{j}^{3}, \\
& \Sigma_{i, j}^{99}=-X_{i}-G_{i}^{3}-G_{i}^{3^{T}} .
\end{aligned}
$$

Theorem 5. The system (1)-(2) is robustly stable if there exist positive definite symmetric matrices $P_{i}, Q_{i}, R_{i}, S_{i}, T_{i}, U_{i}$, $V_{i}, W_{i}, X_{i}, Y_{i}$, and $Z_{i}$, any appropriate dimensional matrices $M_{i}, N_{i}, K_{i}, L_{i}, C_{i}^{j}, D_{i}^{j}, E_{i}^{j}, G_{i}^{j}$, and $L_{i}^{j}, j=1,2,3, i=1,2, \ldots, N$ satisfying the following LMIs:

$$
\begin{gathered}
\prod_{i, i}<-I, \quad i=1,2, \ldots, N \\
\prod_{i, j}+\prod_{j, i}<\frac{2}{N-1} I, \quad i=1, \ldots, N-1, \quad j=i+1, \ldots, N
\end{gathered}
$$$$
\left[\begin{array}{ll}
M_{i} & N_{i} \\
N_{i}^{T} & Y_{i}
\end{array}\right] \geq 0, \quad i=1,2, \ldots, N,
$$

$$
\left[\begin{array}{cc}
K_{i} & L_{i} \\
L_{i}^{T} & Z_{i}
\end{array}\right] \geq 0, \quad i=1,2, \ldots, N .
$$

Proof. Consider the following parameter-dependent Lyapunov-Krasovskii function for the system (9) of the form

$$
V(k)=\sum_{i=1}^{5} V_{i}(k)
$$

where

$$
\begin{aligned}
V_{1}(k)= & x^{T}(k) P(\alpha) x(k) \\
V_{2}(k)= & \sum_{i=k-h_{1}}^{k-1} x^{T}(i) Q(\alpha) x(i)+\sum_{i=k-h_{2}}^{k-1} x^{T}(i) R(\alpha) x(i) \\
& +\sum_{i=k-h_{2}}^{k-h_{1}-1} x^{T}(i) S(\alpha) x(i)+\sum_{i=k-h(k)}^{k-1} x^{T}(i) T(\alpha) x(i),
\end{aligned}
$$




$$
\begin{aligned}
V_{3}(k)= & h_{1} \sum_{j=-h_{1}}^{-1} \sum_{i=k+j}^{k-1} y^{T}(i) U(\alpha) y(i) \\
& +h_{2} \sum_{j=-h_{2}}^{-1} \sum_{i=k+j}^{k-1} y^{T}(i) V(\alpha) y(i) \\
& +h_{2} \sum_{j=-h_{2}}^{-1} \sum_{i=k+j}^{k-1} y^{T}(i) W(\alpha) y(i), \\
V_{4}(k)= & \rho \sum_{j=-h_{2}} \sum_{i=k+j}^{k-1} y^{T}(i) X(\alpha) y(i), \\
V_{5}(k)= & \sum_{j=-h_{1}+1}^{0} \sum_{i=k-1+j}^{k-1} y^{T}(i) Y(\alpha) y(i) \\
& +\sum_{j=-h_{2}+1}^{0} \sum_{i=k-1+j}^{k-1} y^{T}(i) Z(\alpha) y(i) .
\end{aligned}
$$

Evaluating the forward deference of $V(k)$, it is defined as

$$
\Delta V(k)=V(k+1)-V(k) .
$$

Let us define, for $i=1,2, \ldots, 5$,

$$
\Delta V_{i}(k)=V_{i}(k+1)-V_{i}(k) .
$$

Then along the solution of the system (9), we obtain

$$
\begin{aligned}
& \Delta V_{1}(k)= V_{1}(k+1)-V_{1}(k) \\
&= x^{T}(k+1) P(\alpha) x(k+1)-x^{T}(k) P(\alpha) x(k) \\
&=2 x^{T}(k) P(\alpha) y(k)+y^{T}(k) P(\alpha) y(k) \\
&+2 x^{T}(k) L_{1}^{T}(\alpha)[-y(k)+[A(\alpha)+B(\alpha)-I] \\
& \\
&\left.\times x(k)-B(\alpha) \sum_{i=k-h(k)}^{k-1} y(i)\right] \\
&+2 y^{T}(k) L_{2}^{T}(\alpha)[-y(k)+[A(\alpha)+B(\alpha)-I] \\
&\left.\quad \times x(k)-B(\alpha) \sum_{i=k-h(k)}^{k-1} y(i)\right]
\end{aligned}
$$

$$
\begin{array}{r}
+2 \sum_{i=k-h(k)}^{k-1} y(i)^{T} L_{3}^{T}(\alpha)[ \\
-y(k)+[A(\alpha)+B(\alpha)-I] \\
\left.\times x(k)-B(\alpha) \sum_{i=k-h(k)}^{k-1} y(i)\right],
\end{array}
$$$$
\Delta V_{2}(k)=V_{2}(k+1)-V_{2}(k)
$$$$
=x^{T}(k) Q(\alpha) x(k)-x^{T}\left(k-h_{1}\right) Q(\alpha) x\left(k-h_{1}\right)
$$$$
+x^{T}(k) R(\alpha) x(k)-x^{T}\left(k-h_{2}\right) R(\alpha) x\left(k-h_{2}\right)
$$$$
+x^{T}\left(k-h_{1}\right) S(\alpha) x\left(k-h_{1}\right)-x^{T}\left(k-h_{2}\right)
$$$$
\times S(\alpha) x\left(k-h_{2}\right)+x^{T}(k) T(\alpha) x(k)
$$$$
-x^{T}(k-h(k)) T(\alpha) x(k-h(k)) .
$$

\section{From Proposition 4, we have}

$$
\begin{aligned}
& \Delta V_{3}(k)=V_{3}(k+1)-V_{3}(k) \\
& =h_{1}^{2} y^{T}(k) U(\alpha) y(k)-h_{1} \sum_{i=k-h_{1}}^{k-1} y^{T}(i) U(\alpha) y(i) \\
& +h_{2}^{2} y^{T}(k) V(\alpha) y(k)-h_{2} \sum_{i=k-h_{2}}^{k-1} y^{T}(i) V(\alpha) y(i) \\
& +h_{2}^{2} y^{T}(k) W(\alpha) y(k)-h_{2} \sum_{i=k-h_{2}}^{k-1} y^{T}(i) W(\alpha) y(i) \\
& \leq h_{1}^{2} y^{T}(k) U(\alpha) y(k)-\left(\sum_{i=k-h_{1}}^{k-1} y(i)\right)^{T} \\
& \times U(\alpha)\left(\sum_{i=k-h_{1}}^{k-1} y(i)\right)+h_{2}^{2} y^{T}(k) V(\alpha) y(k) \\
& -\left(\sum_{i=k-h_{2}}^{k-1} y(i)\right)^{T} V(\alpha)\left(\sum_{i=k-h_{2}}^{k-1} y(i)\right) \\
& +h_{2}^{2} y^{T}(k) W(\alpha) y(k)-\left(\sum_{i=k-h(k)}^{k-1} y(i)\right)^{T} \\
& \times W(\alpha)\left(\sum_{i=k-h(k)}^{k-1} y(i)\right) \\
& \Delta V_{4}(k)=V_{4}(k+1)-V_{4}(k) \\
& =\rho^{2} y^{T}(k) X(\alpha) y(k)-\rho \sum_{i=k-h_{2}}^{k-h_{1}-1} y^{T}(i) X(\alpha) y(i)
\end{aligned}
$$




$$
\begin{array}{lc}
\leq \rho^{2} y^{T}(k) X(\alpha) y(k)-\left(\sum_{i=k-h_{2}}^{k-h_{1}-1} y(i)\right)^{T} X(\alpha) & \times \sum_{i=-h_{1}+1}^{0} y(k-1+i) \\
\times\left(\sum_{i=k-h_{2}}^{k-h_{1}-1} y(i)\right), & =h_{1} x^{T}(k) M(\alpha) x(k)+2 x^{T}(k) N(\alpha) x(k) \\
& -2 x^{T}(k) N(\alpha) x\left(k-h_{1}\right)
\end{array}
$$

$\Delta V_{5}(k)=V_{5}(k+1)-V_{5}(k)$

$$
=h_{1} y^{T}(k) Y(\alpha) y(k)-\sum_{i=-h_{1}+1}^{0} y^{T}(k-1+i) Y(\alpha)
$$$$
\times y(k-1+i)
$$$$
+h_{2} y^{T}(k) Z(\alpha) y(k)-\sum_{i=-h_{2}+1}^{0} y^{T}(k-1+i) Z(\alpha)
$$$$
\times y(k-1+i) \text {. }
$$

We can show that

$$
\begin{aligned}
& 2 x^{T}(k) N(\alpha) \sum_{i=-h_{1}+1}^{0} y(k-1+i) \\
& +\sum_{i=-h_{1}+1}^{0} y^{T}(k-1+i) Y(\alpha) y(k-1+i)+h_{1} x^{T}(k) M(\alpha) x(k) \\
& =\sum_{i=-h_{1}+1}^{0}\left[\begin{array}{c}
x(k) \\
y-1+i)
\end{array}\right]^{T}\left[\begin{array}{cc}
M(\alpha) & N(\alpha) \\
N^{T}(\alpha) & Y(\alpha)
\end{array}\right] \\
& \quad \times\left[\begin{array}{c}
x(k) \\
y(k-1+i)
\end{array}\right] \geq 0 .
\end{aligned}
$$

It is easy to see that

$$
\begin{aligned}
& 2 x^{T}(k) L(\alpha) \sum_{i=-h_{1}+1}^{0} y(k-1+i) \\
& +\sum_{i=-h_{2}+1}^{0} y^{T}(k-1+i) Z(\alpha) y(k-1+i)+h_{2} x^{T}(k) K(\alpha) x(k) \\
& =\sum_{i=-h_{2}+1}^{0}\left[\begin{array}{c}
x(k) \\
y(k-1+i)
\end{array}\right]^{T}\left[\begin{array}{cc}
K(\alpha) & L(\alpha) \\
L^{T}(\alpha) & Z(\alpha)
\end{array}\right] \\
& \quad \times\left[\begin{array}{c}
x(k) \\
y(k-1+i)
\end{array}\right] \geq 0 .
\end{aligned}
$$

By (22) and (23), we can obtain

$$
\begin{aligned}
& -\sum_{i=-h_{1}+1}^{0} y^{T}(k-1+i) Y(\alpha) y(k-1+i) \\
& \quad \leq h_{1} x^{T}(k) M(\alpha) x(k)+2 x^{T}(k) N(\alpha)
\end{aligned}
$$

and we conclude that

$$
\begin{aligned}
& -\sum_{i=-h_{2}+1}^{0} y^{T}(k-1+i) Z(\alpha) y(k-1+i) \\
& \leq h_{2} x^{T}(k) K(\alpha) x(k)+2 x^{T}(k) L(\alpha) \\
& \quad \times \sum_{i=-h_{2}+1}^{0} y(k-1+i) \\
& =h_{2} x^{T}(k) K(\alpha) x(k)+2 x^{T}(k) L(\alpha) x(k) \\
& \quad-2 x^{T}(k) L(\alpha) x\left(k-h_{2}\right) .
\end{aligned}
$$

It is obvious that

$$
\begin{gathered}
\Upsilon \equiv x(k)-x\left(k-h_{1}\right)-\sum_{i=k-h_{1}}^{k-1} y(i)=0, \\
\Phi \equiv x(k)-x\left(k-h_{2}\right)-\sum_{i=k-h_{2}}^{k-1} y(i)=0, \\
\Psi \equiv x(k)-x(k-h(k))-\sum_{i=k-h(k)}^{k-1} y(i)=0, \\
\Omega \equiv x\left(k-h_{1}\right)-x\left(k-h_{2}\right)-\sum_{i=k-h_{2}}^{k-h_{1}-1} y(i)=0 .
\end{gathered}
$$

The following equations are true for any polytopic matrices with appropriate dimensions:

$$
\begin{aligned}
& {\left[2 x^{T}(k) C_{1}^{T}(\alpha)+2 x^{T}\left(k-h_{1}\right) C_{2}^{T}(\alpha)\right.} \\
& \left.+2\left(\sum_{i=k-h_{1}}^{k-1} y(i)\right)^{T} C_{3}^{T}(\alpha)\right] \times \Upsilon=0, \\
& {\left[2 x^{T}(k) D_{1}^{T}(\alpha)+2 x^{T}\left(k-h_{2}\right) D_{2}^{T}(\alpha)\right.} \\
& \left.+2\left(\sum_{i=k-h_{2}}^{k-1} y(i)\right)^{T} D_{3}^{T}(\alpha)\right] \times \Phi=0,
\end{aligned}
$$




$$
\begin{aligned}
& {\left[2 x^{T}(k) E_{1}^{T}(\alpha)+2 x^{T}(k-h(k)) E_{2}^{T}(\alpha)\right.} \\
& \left.+2\left(\sum_{i=k-h(k)}^{k-1} y(i)\right)^{T} E_{3}^{T}(\alpha)\right] \times \Psi=0, \\
& {\left[2 x^{T}\left(k-h_{1}\right) G_{1}^{T}(\alpha)+2 x^{T}\left(k-h_{2}\right) G_{2}^{T}(\alpha)\right.} \\
& \left.+2\left(\sum_{i=k-h_{2}}^{k-h_{1}-1} y(i)\right)^{T} G_{3}^{T}(\alpha)\right] \times \Omega=0 .
\end{aligned}
$$

It follows from (18)-(30) that

$$
\Delta V(k) \leq \xi^{T}(k) \sum_{i=1}^{N} \sum_{j=1}^{N} \alpha_{i} \alpha_{j} \prod_{i, j} \xi(k)
$$

where $\xi^{T}(k)=\left[x(k)^{T} y(k)^{T} x\left(k-h_{1}\right)^{T} x\left(k-h_{2}\right)^{T} x(k-h(k))^{T}\right.$ $\left.\sum_{i=k-h_{1}}^{k-1} y^{T}(i) \quad \sum_{i=k-h_{2}}^{k-1} y^{T}(i) \quad \sum_{i=k-h(k)}^{k-1} y(i)^{T} \quad \sum_{i=k-h_{2}}^{k-h_{1}-1} y^{T}(i)\right]$ and $\prod_{i, j}$ is defined in (10). Due to the fact that $\sum_{i=1}^{N} \alpha_{i}=1$, we obtain the following identities:

$$
\begin{aligned}
& \sum_{i=1}^{N} \sum_{j=1}^{N} \alpha_{i} \alpha_{j} \prod_{i, j}=\sum_{i=1}^{N} \alpha^{2} \prod_{i, i}+\sum_{i=1}^{N-1} \sum_{j=i+1}^{N} \alpha_{i} \alpha_{i}\left[\prod_{i, j}+\prod_{j, i}\right], \\
& (N-1) \sum_{i=1}^{N} \alpha_{i}^{2}-2 \sum_{i=1}^{N-1} \sum_{j=i+1}^{N} \alpha_{i} \alpha_{j}=\sum_{i=1}^{N-1} \sum_{j=i+1}^{N}\left[\alpha_{i}-\alpha_{j}\right]^{2} \geq 0
\end{aligned}
$$

By (31)-(33), if the conditions (12)-(15) are true, then

$$
\Delta V(k)<-\omega\|x\|^{2}
$$

where $\omega>0$. This means that the system (1)-(2) is robustly stable. The proof of the theorem is complete.

If $A(\alpha)=A$ and $B(\alpha)=B$ when $A, B \in M^{n \times n}$ then the system (1)-(2) reduces to the following system:

$$
\begin{aligned}
& x(k+1)=A x(k)+B x(k-h(k)), \\
& x(s)=\phi(s), \quad s \in\left\{-h_{2}, \ldots,-1,0\right\} .
\end{aligned}
$$

Take the Lyapunov-Krasovskii functional as (16), where $P(\alpha)=P, Q(\alpha)=Q, R(\alpha)=R, S(\alpha)=S, T(\alpha)=T, U(\alpha)=$ $U, V(\alpha)=V, W(\alpha)=W, X(\alpha)=X, Y(\alpha)=Y$, and $Z(\alpha)=Z$ when $P, Q, R, S, T, U, V, W, X, Y, Z \in M^{n \times n}$. Moreover, let us set polytopic matrices with appropriate dimensions of the forms $C_{j}(\alpha)=C^{j}, D_{j}(\alpha)=D^{j}, E_{j}(\alpha)=E^{j}, G_{j}(\alpha)=G^{j}$, and $L_{j}(\alpha)=L^{j}$ when $C^{j}, D^{j}, E^{j}, G^{j}, L^{j} \in M^{n \times n}, j=1,2,3$.
According to Theorem 5, we have Corollary 6 for the delaydependent asymptotically stability criterion of the system (35)-(36). We introduce the following notations for later use:

$$
\Pi=\left[\begin{array}{ccccccccc}
\Sigma^{11} & \Sigma^{12} & \Sigma^{13} & \Sigma^{14} & \Sigma^{15} & \Sigma^{16} & \Sigma^{17} & \Sigma^{18} & 0 \\
* & \Sigma^{22} & 0 & 0 & 0 & 0 & 0 & \Sigma^{28} & 0 \\
* & * & \Sigma^{33} & \Sigma^{34} & 0 & \Sigma^{36} & 0 & 0 & \Sigma^{18} \\
* & * & * & \Sigma^{44} & 0 & 0 & \Sigma^{47} & 0 & \Sigma^{18} \\
* & * & * & * & \Sigma^{55} & 0 & 0 & \Sigma^{18} & 0 \\
* & * & * & * & * & \Sigma^{66} & 0 & 0 & 0 \\
* & * & * & * & * & * & \Sigma^{77} & 0 & 0 \\
* & * & * & * & * & * & * & \Sigma^{88} & 0 \\
* & * & * & * & * & * & * & * & \Sigma^{99}
\end{array}\right],
$$

where

$$
\begin{aligned}
& \Sigma^{11}=Q+R+T+h_{1} M+N+N^{T}+h_{2} K+L+L^{T} \\
& +C^{1}+C^{1^{T}}+D^{1}+D^{1^{T}}+E^{1}+E^{1^{T}}+L^{2^{T}} A \\
& +A^{T} L^{2}+L^{2^{T}} B+B^{T} L^{2}-L^{2}-L^{2^{T}}, \\
& \Sigma^{12}=A^{T} L^{1}+B^{T} L^{1}-L^{1}-L^{2^{T}}+P, \\
& \Sigma^{13}=C^{2}-N-C^{1^{T}}, \quad \Sigma^{14}=D^{2}-L-D^{1^{T}}, \\
& \Sigma^{15}=-E^{1^{T}}+E^{2}, \quad \Sigma^{16}=-C^{1^{T}}+C^{3}, \\
& \Sigma^{17}=-D^{1^{T}}+D^{3} \\
& \Sigma^{18}=-E^{1^{T}}+E^{3}-L^{2^{T}} B+A^{T} L^{3}+B^{T} L^{3}-L^{3}, \\
& \Sigma^{22}=P+h_{1}^{2} U+h_{2}^{2} V+h_{2}^{2} W+\rho^{2} X \\
& +h_{1} Y+h_{2} Z-L^{1}-L^{1^{T}} \text {, } \\
& \Sigma^{28}=-L^{1^{T}} B-L^{3}, \\
& \Sigma^{33}=-Q+S-C^{2}-C^{2^{T}}+G^{1}+G^{1^{T}}, \quad \Sigma^{34}=G^{2}-G^{1^{T}}, \\
& \Sigma^{36}=-C^{3}-C^{2^{T}}, \quad \Sigma^{39}=G^{3}-G^{1^{T}}, \\
& \Sigma^{44}=-R-S-D^{2}-D^{2^{T}}-G^{2}-G^{2^{T}}, \\
& \Sigma^{47}=-D^{3}-D^{2^{T}} \\
& \Sigma^{49}=-G^{3}-G^{2^{T}}, \quad \Sigma^{55}=-T-E^{2}-E^{2^{T}}, \\
& \Sigma^{58}=-E^{3}-E^{2^{T}}, \\
& \Sigma^{66}=-U-C^{3}-C^{3^{T}}, \quad \Sigma^{77}=-V-D^{3}-D^{3^{T}}, \\
& \Sigma^{88}=-W-E^{3}-E^{3^{T}}-L^{3^{T}} B-B^{T} L^{3}, \\
& \Sigma^{99}=-X-G^{3}-G^{3^{T}}
\end{aligned}
$$




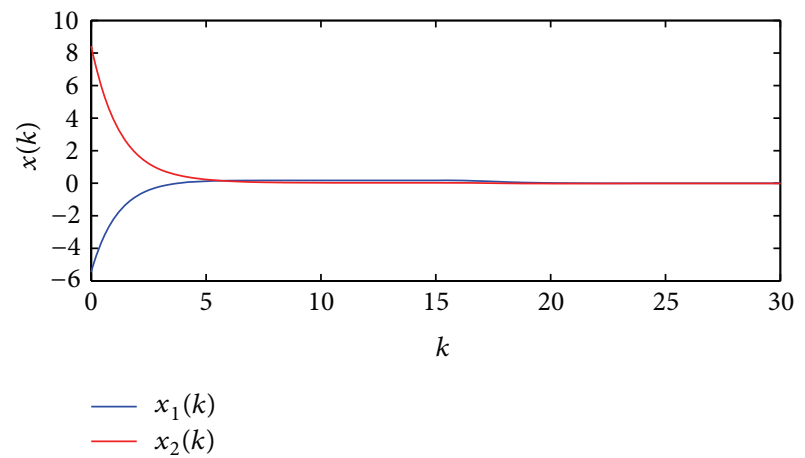

Figure 1: The Simulation solution of the states $x_{1}(k)$ and $x_{2}(k)$ in Example 7 for LPD discrete-time delayed system with initial conditions $x_{1}(k)=-5.5$ and $x_{2}(k)=8.5, k=-16, \ldots,-2,-1,0$ and $\alpha_{1}=1 / 3, \alpha_{2}=2 / 3$ by using method of Runge-Kutta order 4 $(h=0.01)$ with Matlab.

Corollary 6. The system (35)-(36) is asymptotically stable if there exist positive definite symmetric matrices $P, Q, R, S, T$, $U, V, W, X, Y$, and $Z$, any appropriate dimensional matrices $M, N, K, L, C^{j}, D^{j}, E^{j}, G^{j}$, and $L^{j}, j=1,2,3$, satisfying the following LMIs:

$$
\begin{gathered}
\prod<0, \\
{\left[\begin{array}{cc}
M & N \\
N^{T} & Y
\end{array}\right] \geq 0,} \\
{\left[\begin{array}{ll}
K & L \\
L^{T} & Z
\end{array}\right] \geq 0 .}
\end{gathered}
$$

\section{Numerical Examples}

Example 7. Consider the following LPD discrete-time system with interval time-varying delays (1)-(2) with

$$
\begin{array}{cc}
A_{1}=\left[\begin{array}{cc}
0.80 & 0 \\
0.01 & 0.60
\end{array}\right], & A_{2}=\left[\begin{array}{cc}
0.90 & 0 \\
0.05 & 0.90
\end{array}\right], \\
B_{1}=\left[\begin{array}{cc}
0.10 & 0 \\
0.20 & 0.10
\end{array}\right], & B_{2}=\left[\begin{array}{cc}
-0.10 & 0 \\
-0.20 & -0.10
\end{array}\right],
\end{array}
$$

$h(k)=2+14 \cos ^{2}(k \pi / 2)$ with initial condition $\phi(k)=\left[\begin{array}{c}-5.5 \\ 8.5\end{array}\right]$, $k \in[-16,0]$. The numerical solutions $x_{1}(k)$ and $x_{2}(k)$ of (1)(2) with (40) are plotted in Figure 1.

Solution. By using the LMI Toolbox in MATLAB (with accuracy 0.01 ) and conditions (12)-(15) of Theorem 5, this system is robustly stable for discrete delay time satisfying $h_{1}=2, h_{2}=16$, and

$$
\begin{aligned}
& P_{1}=10^{6} \times\left[\begin{array}{cc}
4.3970 & -0.4104 \\
-0.4104 & 0.3531
\end{array}\right], \\
& P_{2}=10^{5} \times\left[\begin{array}{cc}
5.2274 & -0.0613 \\
-0.0613 & 0.3488
\end{array}\right] \text {, } \\
& Q_{1}=10^{4} \times\left[\begin{array}{cc}
4.9438 & -1.3988 \\
-1.3988 & 1.3372
\end{array}\right] \text {, } \\
& Q_{2}=10^{4} \times\left[\begin{array}{cc}
1.6042 & -0.3173 \\
-0.3173 & 0.1431
\end{array}\right] \text {, } \\
& R_{1}=10^{4} \times\left[\begin{array}{cc}
1.8144 & -0.5737 \\
-0.5737 & 0.4286
\end{array}\right] \text {, } \\
& R_{2}=10^{3} \times\left[\begin{array}{cc}
4.2669 & -0.8605 \\
-0.8605 & 0.3167
\end{array}\right] \text {, } \\
& S_{1}=10^{4} \times\left[\begin{array}{cc}
2.3161 & -0.7409 \\
-0.7409 & 0.5638
\end{array}\right] \text {, } \\
& S_{2}=10^{3} \times\left[\begin{array}{cc}
5.5557 & -1.1238 \\
-1.1238 & 0.4007
\end{array}\right] \text {, } \\
& T_{1}=10^{5} \times\left[\begin{array}{cc}
2.2862 & -0.3474 \\
-0.3474 & 0.2764
\end{array}\right] \text {, } \\
& T_{2}=10^{4} \times\left[\begin{array}{ll}
5.0867 & 0.3198 \\
0.3198 & 0.2709
\end{array}\right] \text {, } \\
& U_{1}=10^{4} \times\left[\begin{array}{cc}
9.6266 & -0.3736 \\
-0.3736 & 2.6793
\end{array}\right] \text {, } \\
& U_{2}=10^{4} \times\left[\begin{array}{cc}
6.7104 & -1.2896 \\
-1.2896 & 0.6493
\end{array}\right] \text {, } \\
& V_{1}=10^{3} \times\left[\begin{array}{cc}
2.6354 & -0.1810 \\
-0.1810 & 0.2286
\end{array}\right] \text {, } \\
& V_{2}=10^{3} \times\left[\begin{array}{cc}
1.1098 & -0.2116 \\
-0.2116 & 0.0795
\end{array}\right] \text {, } \\
& W_{1}=10^{4} \times\left[\begin{array}{ll}
1.2581 & 0.0031 \\
0.0031 & 0.0279
\end{array}\right] \text {, } \\
& W_{2}=10^{3} \times\left[\begin{array}{ll}
9.8860 & 0.9947 \\
0.9947 & 0.4654
\end{array}\right] \text {, } \\
& X_{1}=10^{3} \times\left[\begin{array}{cc}
3.6535 & -0.2316 \\
-0.2316 & 0.3050
\end{array}\right] \text {, } \\
& X_{2}=10^{3} \times\left[\begin{array}{cc}
1.5049 & -0.2858 \\
-0.2858 & 0.1068
\end{array}\right] \text {, } \\
& Y_{1}=10^{5} \times\left[\begin{array}{cc}
1.0677 & -0.0668 \\
-0.0668 & 0.3783
\end{array}\right] \text {, } \\
& Y_{2}=10^{4} \times\left[\begin{array}{cc}
8.4989 & -1.5451 \\
-1.5451 & 1.0863
\end{array}\right] \text {, }
\end{aligned}
$$




$$
\begin{aligned}
& Z_{1}=10^{4} \times\left[\begin{array}{cc}
4.8154 & -0.3746 \\
-0.3746 & 0.6475
\end{array}\right] \\
& Z_{2}=10^{4} \times\left[\begin{array}{cc}
2.5697 & -0.4901 \\
-0.4901 & 0.2043
\end{array}\right] \text {, } \\
& C_{1}^{1}=10^{4} \times\left[\begin{array}{ll}
-1.5983 & -0.1755 \\
-0.1755 & -1.6231
\end{array}\right] \text {, } \\
& C_{2}^{1}=10^{4} \times\left[\begin{array}{cc}
-2.1448 & 0.1127 \\
0.1127 & -1.6904
\end{array}\right] \text {, } \\
& C_{1}^{2}=10^{4} \times\left[\begin{array}{ll}
1.2837 & 0.1710 \\
0.1710 & 1.5002
\end{array}\right] \text {, } \\
& C_{2}^{2}=10^{4} \times\left[\begin{array}{cc}
1.6947 & -0.0260 \\
-0.0260 & 1.6512
\end{array}\right] \text {, } \\
& C_{1}^{3}=10^{3} \times\left[\begin{array}{cc}
-7.3049 & 0.2491 \\
0.2491 & 9.7027
\end{array}\right] \text {, } \\
& C_{2}^{3}=10^{4} \times\left[\begin{array}{cc}
-0.1374 & 0.3389 \\
0.3389 & 1.4742
\end{array}\right] \text {, } \\
& D_{1}^{1}=10^{4} \times\left[\begin{array}{ll}
-0.9350 & -0.1962 \\
-0.1962 & -1.4853
\end{array}\right] \text {, } \\
& D_{2}^{1}=10^{4} \times\left[\begin{array}{cc}
-1.7099 & 0.0229 \\
0.0229 & -1.6489
\end{array}\right] \text {, } \\
& D_{1}^{2}=10^{4} \times\left[\begin{array}{ll}
0.5714 & 0.2950 \\
0.2950 & 1.3754
\end{array}\right] \text {, } \\
& D_{2}^{2}=10^{4} \times\left[\begin{array}{ll}
1.3928 & 0.0393 \\
0.0393 & 1.6269
\end{array}\right] \text {, } \\
& D_{1}^{3}=10^{4} \times\left[\begin{array}{cc}
1.9066 & -0.0891 \\
-0.0891 & 1.7055
\end{array}\right] \text {, } \\
& D_{2}^{3}=10^{4} \times\left[\begin{array}{cc}
1.7454 & -0.0290 \\
-0.0290 & 1.6519
\end{array}\right] \text {, } \\
& E_{1}^{1}=10^{4} \times\left[\begin{array}{ll}
-4.2910 & -1.6701 \\
-1.6701 & -1.5104
\end{array}\right] \text {, } \\
& E_{2}^{1}=10^{4} \times\left[\begin{array}{ll}
-3.7600 & -0.2382 \\
-0.2382 & -1.7532
\end{array}\right] \text {, } \\
& E_{1}^{2}=10^{4} \times\left[\begin{array}{cc}
-7.0237 & 0.5763 \\
0.5763 & 0.8408
\end{array}\right] \text {, } \\
& E_{2}^{2}=10^{4} \times\left[\begin{array}{cc}
-0.7534 & -0.1269 \\
-0.1269 & 1.4957
\end{array}\right] \text {, } \\
& E_{1}^{3}=10^{5} \times\left[\begin{array}{cc}
1.0553 & -0.0488 \\
-0.0488 & 0.2403
\end{array}\right] \text {, } \\
& E_{2}^{3}=10^{4} \times\left[\begin{array}{ll}
3.8446 & 0.2721 \\
0.2721 & 1.7426
\end{array}\right], \\
& G_{1}^{1}=10^{4} \times\left[\begin{array}{ll}
-1.1174 & -0.1492 \\
-0.1492 & -1.4929
\end{array}\right] \text {, }
\end{aligned}
$$

$$
\begin{aligned}
& G_{2}^{1}=10^{4} \times\left[\begin{array}{ll}
-1.6145 & -0.0032 \\
-0.0032 & -1.6397
\end{array}\right], \\
& G_{1}^{2}=10^{4} \times\left[\begin{array}{ll}
0.6849 & 0.2738 \\
0.2738 & 1.3873
\end{array}\right], \\
& G_{2}^{2}=10^{4} \times\left[\begin{array}{ll}
1.3546 & 0.0545 \\
0.0545 & 1.6203
\end{array}\right] \text {, } \\
& G_{1}^{3}=10^{4} \times\left[\begin{array}{cc}
1.8742 & -0.0945 \\
-0.0945 & 1.7071
\end{array}\right] \text {, } \\
& G_{2}^{3}=10^{4} \times\left[\begin{array}{cc}
1.7014 & -0.0146 \\
-0.0146 & 1.6454
\end{array}\right] \text {, } \\
& L_{1}^{1}=10^{6} \times\left[\begin{array}{cc}
5.3779 & -0.2824 \\
-0.2824 & 0.4796
\end{array}\right] \text {, } \\
& L_{2}^{1}=10^{6} \times\left[\begin{array}{ll}
2.3117 & 0.0294 \\
0.0294 & 0.3636
\end{array}\right] \text {, } \\
& L_{1}^{2}=10^{6} \times\left[\begin{array}{cc}
4.0552 & -0.3696 \\
-0.3696 & 0.2311
\end{array}\right] \text {, } \\
& L_{2}^{2}=10^{4} \times\left[\begin{array}{ll}
9.4267 & 0.9110 \\
0.9110 & 7.4877
\end{array}\right] \text {, } \\
& L_{1}^{3}=10^{5} \times\left[\begin{array}{ll}
-2.9313 & -0.2209 \\
-0.2209 & -0.4362
\end{array}\right] \text {, } \\
& L_{2}^{3}=10^{5} \times\left[\begin{array}{cc}
2.1784 & -0.0837 \\
-0.0837 & -0.1597
\end{array}\right] \text {, } \\
& K_{1}=\left[\begin{array}{cc}
897.9190 & -266.0787 \\
-266.0787 & 187.1557
\end{array}\right] \text {, } \\
& K_{2}=\left[\begin{array}{cc}
236.7231 & -47.9750 \\
-47.9750 & 19.2914
\end{array}\right] \text {, } \\
& M_{1}=10^{3} \times\left[\begin{array}{cc}
6.1473 & -1.9404 \\
-1.9404 & 1.4158
\end{array}\right] \text {, } \\
& M_{2}=10^{3} \times\left[\begin{array}{cc}
1.6184 & -0.3310 \\
-0.3310 & 0.1305
\end{array}\right] \text {, } \\
& N_{1}=10^{3} \times\left[\begin{array}{cc}
-5.4598 & 0.9628 \\
0.9628 & -2.2399
\end{array}\right] \text {, } \\
& N_{2}=10^{3} \times\left[\begin{array}{cc}
-3.9963 & 0.7762 \\
0.7762 & -0.4562
\end{array}\right] \text {, } \\
& L_{1}=10^{3} \times\left[\begin{array}{cc}
-2.4982 & 0.3102 \\
0.3102 & -0.3738
\end{array}\right] \text {, } \\
& L_{2}=10^{3} \times\left[\begin{array}{cc}
-1.1912 & 0.2324 \\
0.2324 & -0.0945
\end{array}\right] \text {. }
\end{aligned}
$$

For the given $h_{1}$, Table 1 lists the comparison of the upper bounds delay $h_{2}$ for robust stability of the system (1)-(2) with (40) by the different method. By a conditions in Theorem 5, we can see from Table 1 that our result is superior to those in [22, Theorem 1]. 
TABLE 1: Comparison of the maximum allowed time delay $h_{2}$ for different conditions.

\begin{tabular}{lcccc}
\hline$h_{1}$ & 2 & 4 & 5 & 6 \\
\hline Liu et al. [10] (2006) & 2 & 4 & 5 & 6 \\
Zhang et al. [22] (2010) & 12 & 13 & 14 & 15 \\
Theorem 5 & $>15$ & $>16$ & $>17$ & $>18$ \\
\hline
\end{tabular}

TABLE 2: Comparison of the maximum allowed time delay $h_{2}$ for different conditions.

\begin{tabular}{lcccc}
\hline$h_{1}$ & 7 & 10 & 15 & 20 \\
\hline Fridman and Shaked [6] (2005) & 13 & 15 & 19 & 23 \\
Gao and Chen [1](2007) & 14 & 15 & 18 & 22 \\
Zhang et al. [22] (2010) & 15 & 17 & 21 & 25 \\
Zhang et al. [23] (2011) & 15 & 17 & 21 & 25 \\
Corollary 6 & $>18$ & $>19$ & $>24$ & $>27$ \\
\hline
\end{tabular}

Example 8. Consider the system (35) with

$$
A=\left[\begin{array}{cc}
0.80 & 0 \\
0.05 & 0.90
\end{array}\right], \quad B=\left[\begin{array}{cc}
-0.10 & 0 \\
-0.20 & -0.10
\end{array}\right] \text {. }
$$

For the given $h_{1}$, we calculate the allowable maximum value of $h_{2}$ that guarantees the asymptotic stability of the system (35) with (42). By using different methods, the calculated results are presented in Table 2 . From the table, we can see that Corollary 6 in this paper provides the less conservative results.

\section{Acknowledgments}

This work is supported by National Research Council of Thailand and Khon Kaen University, Thailand (Grant no. kku fmis 121740).

\section{References}

[1] H. Gao and T. Chen, "New results on stability of discrete-time systems with time-varying state delay," Institute of Electrical and Electronics Engineers, vol. 52, no. 2, pp. 328-334, 2007.

[2] T. Botmart and P. Niamsup, "Robust exponential stability and stabilizability of linear parameter dependent systems with delays," Applied Mathematics and Computation, vol. 217, no. 6, pp. 2551-2566, 2010.

[3] B. T. Cui and M. G. Hua, "Robust passive control for uncertain discrete-time systems with time-varying delays," Chaos, Solitons and Fractals, vol. 29, no. 2, pp. 331-341, 2006.

[4] M. Fang, "Delay-dependent stability analysis for discrete singular systems with time-varying delays," Acta Automatica Sinica, vol. 36, no. 5, pp. 751-755, 2010.

[5] E. Fridman and U. Shaked, "Parameter dependent stability and stabilization of uncertain time-delay systems," Institute of Electrical and Electronics Engineers, vol. 48, no. 5, pp. 861-866, 2003.

[6] E. Fridman and U. Shaked, "Stability and guaranteed cost control of uncertain discrete delay systems," International Journal of Control, vol. 78, no. 4, pp. 235-246, 2005.
[7] K. Gu, V. L. Kharitonov, and J. Chen, Stability of Time-Delay Systems, Birkhauser, Berlin, Germany, 2003.

[8] Y. He, M. Wu, J.-H. She, and G.-P. Liu, "Parameter-dependent Lyapunov functional for stability of time-delay systems with polytopic-type uncertainties," Institute of Electrical and Electronics Engineers, vol. 49, no. 5, pp. 828-832, 2004.

[9] X. Jiang, Q. L. Han, and X. Yu, "Stability criteria for linear discrete-time systems with interval-like time-varying delay," in Proceedings of the American Control Conference (ACC '05), pp. 2817-2822, June 2005.

[10] X. G. Liu, R. R. Martin, M. Wu, and M. L. Tang, "Delaydependent robust stabilisation of discrete-time systems with time-varying delay," IEE Proceedings: Control Theory and Applications, vol. 153, no. 6, pp. 689-702, 2006.

[11] W.-J. Mao, "An LMI approach to $D$-stability and $D$-stabilization of linear discrete singular system with state delay," Applied Mathematics and Computation, vol. 218, no. 5, pp. 1694-1704, 2011.

[12] W.-J. Mao and J. Chu, "D-stability and D-stabilization of linear discrete time-delay systems with polytopic uncertainties," Automatica, vol. 45, no. 3, pp. 842-846, 2009.

[13] K. Mukdasai, "Robust exponential stability for LPD discretetime system with interval time-varying delay," Journal of Applied Mathematics, vol. 2012, Article ID 237430, 13 pages, 2012.

[14] K. Mukdasai and P. Niamsup, "Robust stability of discrete-time linear parameter dependent system with delay," Thai Journal of Mathematics (Annual Meeting in Mathematics), vol. 8, no. 4, Special issue, pp. 11-20, 2010.

[15] P. T. Nam, H. M. Hien, and V. N. Phat, "Asymptotic stability of linear state-delayed neutral systems with polytope type uncertainties," Dynamic Systems and Applications, vol. 19, no. 1, pp. 63-72, 2010.

[16] P. Niamsup and V. N. Phat, " $H_{\infty}$ control for nonlinear timevarying delay systems with convex polytopic uncertainties," Nonlinear Analysis: Theory, Methods \& Applications, vol. 72, no. 11, pp. 4254-4263, 2010.

[17] V. N. Phat and P. T. Nam, "Exponential stability and stabilization of uncertain linear time-varying systems using parameter dependent Lyapunov function," International Journal of Control, vol. 80, no. 8, pp. 1333-1341, 2007.

[18] M. De la Sen, "Robust stabilization of a class of polytopic linear time-varying continuous systems under point delays and saturating controls," Applied Mathematics and Computation, vol. 181, no. 1, pp. 73-83, 2006.

[19] S. Udpin and P. Niamsup, "Robust stability of discrete-time LPD neural networks with time-varying delay," Communications in Nonlinear Science and Numerical Simulation, vol. 14, no. 11, pp. 3914-3924, 2009.

[20] Z. G. Wu, P. Shi, H. Su, and J. Chu, "Delay-dependent exponential stability analysis for discrete-time switched neural networks with time-varying delay," Neurocomputing, vol. 74, no. 10, pp. 1626-1631, 2011.

[21] Y. Xia and Y. Jia, "Robust stability functionals of state delayed systems with polytopic type uncertainties via parameterdependent Lyapunov functions," International Journal of Control, vol. 75, no. 16-17, pp. 1427-1434, 2002.

[22] W. Zhang, Q. Y. Xie, X. S. Cai, and Z. Z. Han, "New stability criteria for discrete-time systems with interval time-varying delay and polytopic uncertainty," Latin American Applied Research, vol. 40, no. 2, pp. 119-124, 2010. 
[23] W. Zhang, J. Wang, Y. Liang, and Z. Z. Han, "Improved delayrange-dependent stability criterion for discrete-time systems with interval time-varying delay," Journal of Information and Computational Science, vol. 14, no. 8, pp. 3321-3328, 2011.

[24] W.-A. Zhang and L. Yu, "Stability analysis for discrete-time switched time-delay systems," Automatica, vol. 45, no. 10, pp. 2265-2271, 2009. 


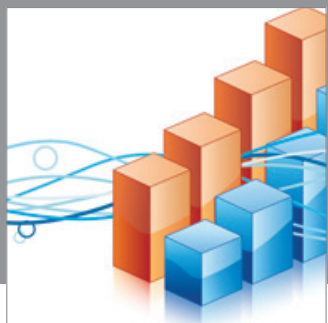

Advances in

Operations Research

mansans

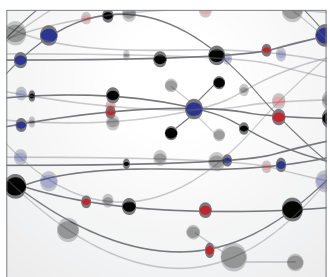

The Scientific World Journal
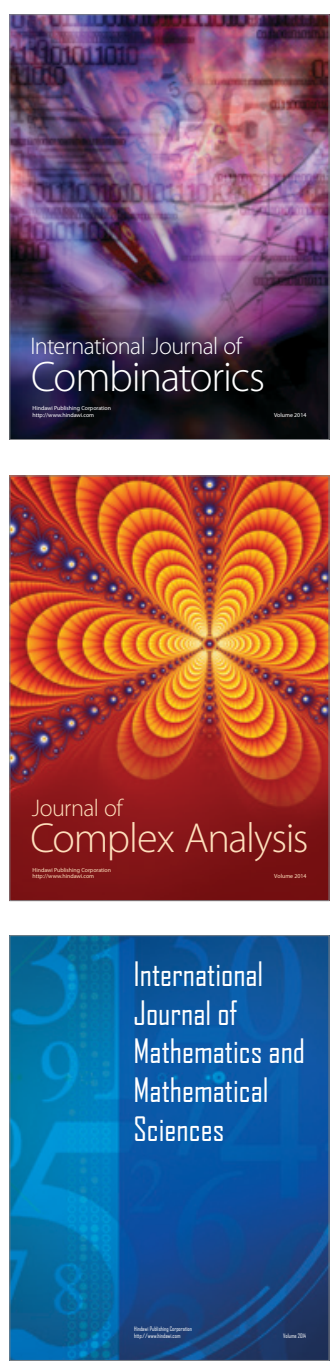
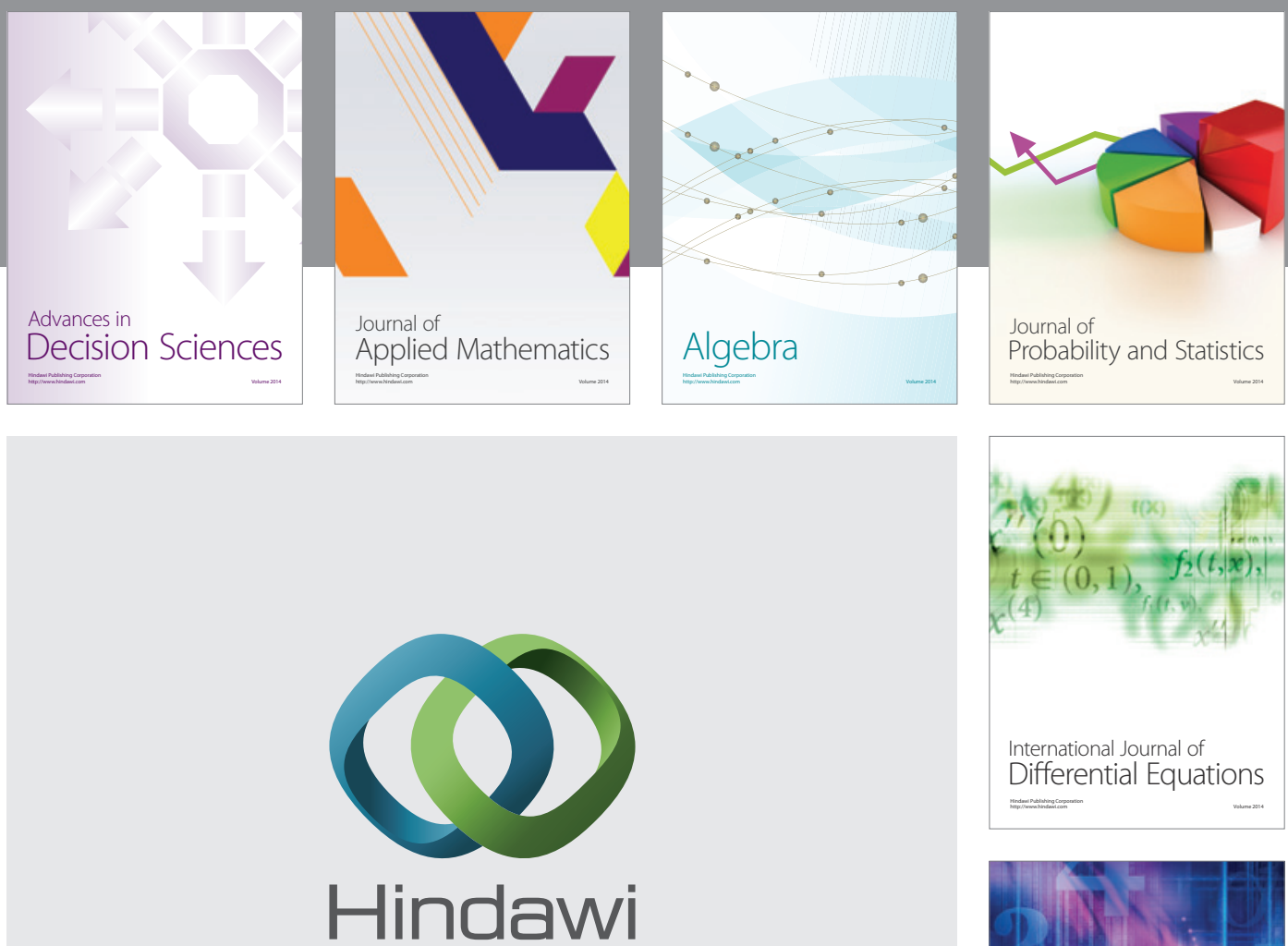

Submit your manuscripts at http://www.hindawi.com
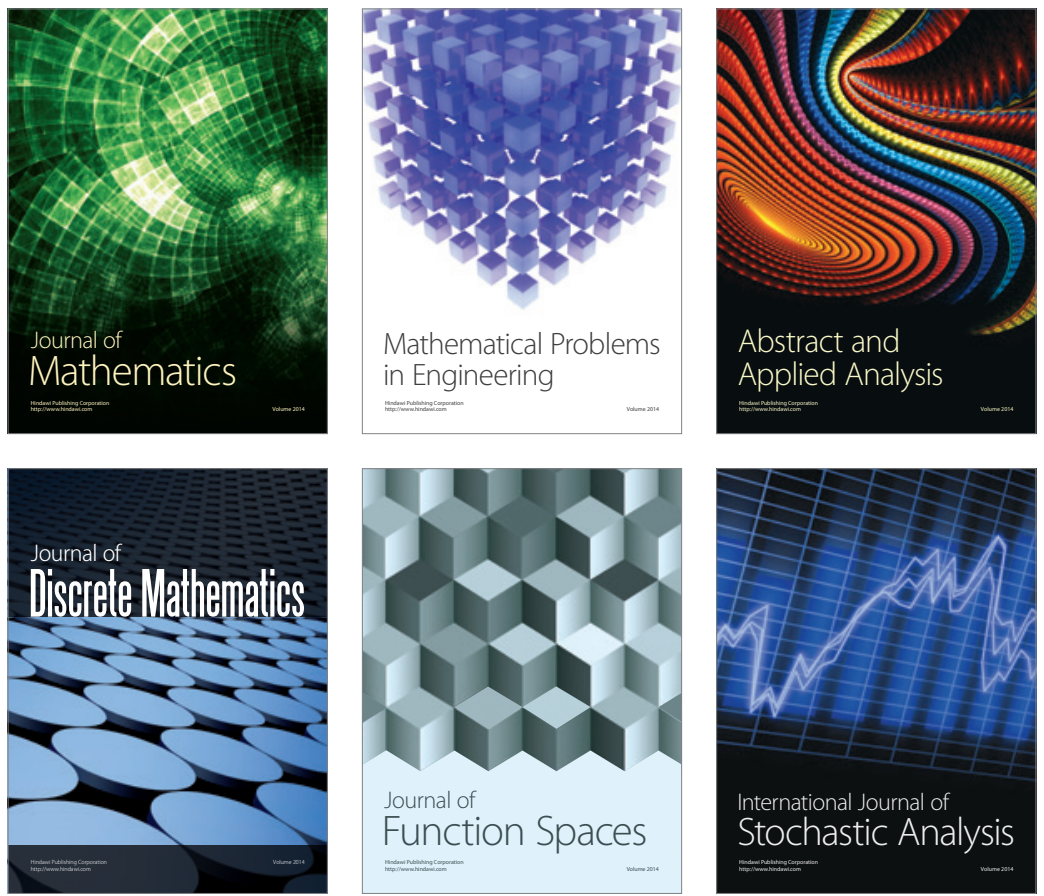

Journal of

Function Spaces

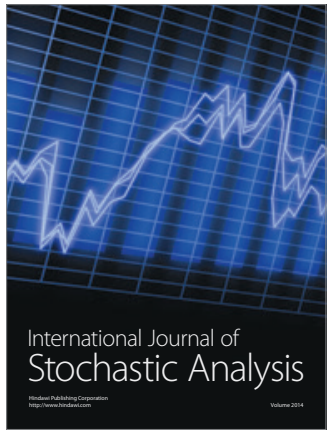

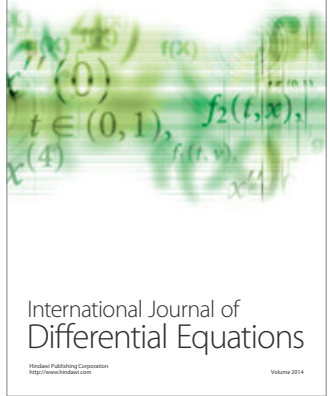
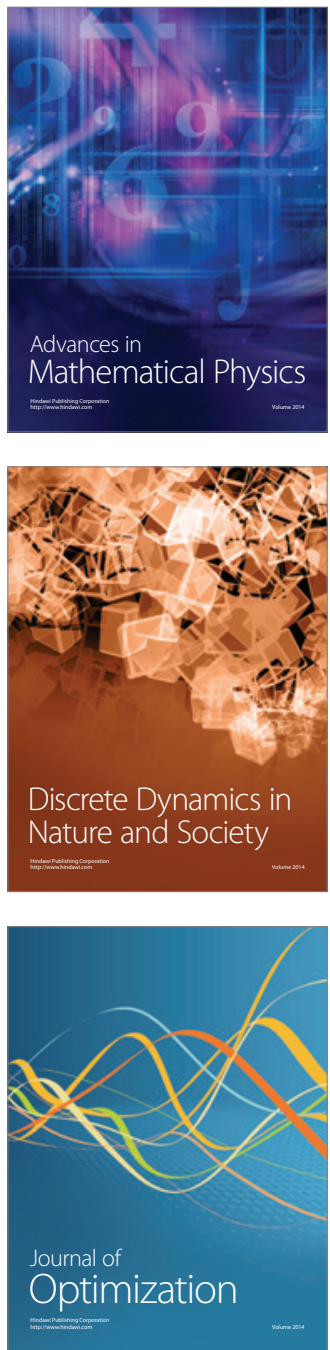